Supplementary Information

\title{
Phytoalexins from the crucifer rutabaga: structures, syntheses, biosyntheses and antifungal activity
}

M. Soledade C. Pedras, ${ }^{*}$ Sabine Montaut and Mojmir Suchy

Department of Chemistry, University of Saskatchewan, 110 Science Place, Saskatoon SK S7N 5C9, Canada

\section{Supplementary Information}

Table of Contents:

Experimental general

Synthesis of compounds $17,18,19,24 a$

Spectral data for compound 9
S1

S1-S4

S4 
Supplementary Information

\section{Experimental}

General. All solvents were HPLC grade and used as such, except for $\mathrm{CH}_{2} \mathrm{Cl}_{2}$ and $\mathrm{CHCl}_{3}$ which were redistilled and THF which was dried over $\mathrm{Na}$ and benzophenone. Flash $\mathrm{CC}$ : C-18 reversed phase silica gel $40 \mu \mathrm{m}$. Analytical HPLC analysis was carried out with a high performance liquid chromatograph equipped with quaternary pump, automatic injector, and photodiode array detector (wavelength range 190-600 nm), degasser, and an ODS column ( $5 \mu \mathrm{m}$ particle size silica, 4.6 i.d. $\times 200 \mathrm{~mm})$, equipped with an in-line filter. Mobile phase: $\mathrm{H}_{2} \mathrm{O}-\mathrm{CH}_{3} \mathrm{CN}, 75: 25$ to $100 \% \mathrm{CH}_{3} \mathrm{CN}$, for 35 min, linear gradient, and flow rate $1.0 \mathrm{~mL} / \mathrm{min}$. Other conditions as previously reported. ${ }^{19}$

1-Boc-2-chloroindole-3-carboxylic acid (17). 1-Boc-2-chloroindole-3-carboxaldehyde ${ }^{13}$ $(16,0.56 \mathrm{~g}, 2 \mathrm{mmol})$ was dissolved in the mixture of $t$-butanol $(10 \mathrm{~mL})$ and 2-methylbut2-ene $(10 \mathrm{~mL})$. A solution of $\mathrm{NaClO}_{2}(1.81 \mathrm{~g}, 20 \mathrm{mmol})$ and $\mathrm{KH}_{2} \mathrm{PO}_{4}(2.04 \mathrm{~g}, 15 \mathrm{mmol})$ in water $(10 \mathrm{~mL})$ was then added and the mixture was vigorously stirred at $\mathrm{rt}$. After $2 \mathrm{~h}$ the organic layer was separated, the aq layer was extracted with $\mathrm{CH}_{2} \mathrm{Cl}_{2}-\mathrm{MeOH}$ (95:5), the combined organic extracts were dried $\left(\mathrm{Na}_{2} \mathrm{SO}_{4}\right)$ and the solvent evaporated under reduced pressure. Crystallization $\left(\mathrm{CH}_{2} \mathrm{Cl}_{2}\right.$-hexane) afforded $0.567 \mathrm{~g}(96 \%)$ of colorless powder. Mp $175-177{ }^{\circ} \mathrm{C}$; HPLC $t_{\mathrm{R}}=19.8 \mathrm{~min} ;{ }^{1} \mathrm{H}$ NMR $\left(500 \mathrm{MHz}, \mathrm{CDCl}_{3}\right): 12.68(\mathrm{br}$ $\mathrm{s}, 1 \mathrm{H}, \mathrm{D}_{2} \mathrm{O}$ exchangeable), $8.25(\mathrm{~m}, 1 \mathrm{H}), 8.04(\mathrm{~m}, 1 \mathrm{H}), 7.38(\mathrm{~m}, 2 \mathrm{H}), 1.74(\mathrm{~s}, 9 \mathrm{H}) ;{ }^{13} \mathrm{C}$ NMR (125 MHz, CDCl 3 ): 168.9 (s), 148.3 (s), 135.2 (s), 132.7 (s), 126.2 (s), 125.5 (d), 124.6 (d), 121.8 (d), 114.6 (d), 109.5 (s), 86.8 (s), 28.3 (q); HREIMS $m / z$ (\% relative abundance) measured: 295.0607 (295.0611 calcd for $\left.\mathrm{C}_{14} \mathrm{H}_{14} \mathrm{ClNO}_{4}\right)$; EIMS $\mathrm{m} / z(\%$ relative abundance): $295[\mathrm{M}]^{+}(6), 221$ (5), 195 (37), 130 (7). FTIR $v_{\max }$ : 2979, 1745, $1683,1532,1445,1308,1206,1150,745 \mathrm{~cm}^{-1}$.

Methyl 1-Boc-2-chloroindole-3-carboxylate (18). 1-Boc-2-chloroindole-3-carboxylic acid $(\mathbf{1 7}, 74 \mathrm{mg}, 0.25 \mathrm{mmol})$ was dissolved in ethereal diazomethane solution $(3 \mathrm{~mL})$ and 
Supplementary Information

stirred for 5 minutes at rt. The excess of diazomethane was quenched with acetic acid and the solvent was evaporated under reduced pressure. The colorless oil (77 $\mathrm{mg}, 100 \%)$ was obtained, sufficiently pure to use in the next step. HPLC $t_{\mathrm{R}}=35.1 \mathrm{~min} ;{ }^{1} \mathrm{H}$ NMR (500 $\left.\mathrm{MHz}, \mathrm{CDCl}_{3}\right): 8.12(\mathrm{~d}, J=8 \mathrm{~Hz}, 1 \mathrm{H}), 8.04(\mathrm{~d}, J=8 \mathrm{~Hz}, 1 \mathrm{H}), 7.35(\mathrm{~m}, 2 \mathrm{H}), 4.00(\mathrm{~s}, 3 \mathrm{H})$, 1.73 (s, 9H); ${ }^{13} \mathrm{C}$ NMR (125 MHz, $\left.\mathrm{CDCl}_{3}\right): 164.0$ (s), 148.5 (s), 135.2 (s), 131.1 (s), 126.1 (s), 125.3 (d), 124.3 (d), 121.5 (d), 114.7 (d), 110.5 (s), 86.5 (s), 51.8 (q), 28.3 (q); HREIMS m/z (\% relative abundance) measured: 309.0765 (309.0768 calcd for $\mathrm{C}_{15} \mathrm{H}_{16} \mathrm{ClNO}_{4}$ ); EIMS m/z (\% relative abundance): 309 [M] $]^{+}$(20), 252 (6), 236 (13), 209 (100), 178 (23), 148 (5), 114 (22). FTIR $v_{\max }$ : 2980, 1749, 1722, 1532, 1446, 1309, 1147, $747 \mathrm{~cm}^{-1}$.

Methyl 1-Boc-2-chloroindole-3-carboxamide (19). Thionyl chloride (30 $\mu \mathrm{L}, 0.45$ mmol) was added to a solution of acid 17 (90 mg, $0.3 \mathrm{mmol})$ in dry THF (3 mL) under Ar atmosphere and the mixture was stirred at $\mathrm{rt}$. After $3 \mathrm{~h}$ the reaction mixture was cooled to $0{ }^{\circ} \mathrm{C}$, a solution of $\mathrm{MeNH}_{2}$ in THF $(2 \mathrm{M}, 4 \mathrm{~mL}, 8 \mathrm{mmol})$ was added and the mixture was stirred for further $20 \mathrm{~min}$ at $0{ }^{\circ} \mathrm{C}$. Then, the reaction mixture was diluted with brine $(30$ $\mathrm{mL})$, extracted with EtOAc, the combined organic extract was dried $\left(\mathrm{Na}_{2} \mathrm{SO}_{4}\right)$, the solvent evaporated under reduced pressure and the residue chromatographed on silica gel (hexane-acetone, 2:1). Evaporation of the solvent afforded a colorless oil (79 $\mathrm{mg}, 85 \%$ ). An analytical sample was obtained by crystallization from $\mathrm{CH}_{2} \mathrm{Cl}_{2}$-hexane. $\mathrm{Mp} 92-94$ ${ }^{\circ} \mathrm{C}$; HPLC $t_{\mathrm{R}}=20.9 \mathrm{~min} ;{ }^{1} \mathrm{H}$ NMR $\left(500 \mathrm{MHz}, \mathrm{CDCl}_{3}\right): 8.18(\mathrm{~d}, J=8 \mathrm{~Hz}, 1 \mathrm{H}), 8.02(\mathrm{~d}, J$ $=8 \mathrm{~Hz}, 1 \mathrm{H}), 7.34(\mathrm{~m}, 2 \mathrm{H}), 6.50$ (br s , 1H, $\mathrm{D}_{2} \mathrm{O}$ exchangeable), $3.08(\mathrm{~d}, J=5 \mathrm{~Hz}, 3 \mathrm{H})$, 1.72 (s, 9H); ${ }^{13} \mathrm{C}$ NMR (125 MHz, $\left.\mathrm{CDCl}_{3}\right): 163.6$ (s), 148.7 (s), 135.2 (s), 126.8 (s), 125.5 (d), 124.2 (d), 123.8 (s), 121.5 (d), 114.8 (s), 114.7 (d), 86.2 (s), 28.3 (q), 26.6 (q); HREIMS $m / z$ (\% relative abundance) measured: 308.0923 (309.0928 calcd for $\mathrm{C}_{15} \mathrm{H}_{17} \mathrm{ClN}_{2} \mathrm{O}_{3}$ ); EIMS m/z (\% relative abundance): $308[\mathrm{M}]^{+}$(6), 208 (37), 178 (29), 149 (15). FTIR $v_{\max }: 3291,2977,1743,1643,1546,1447,1309,1154,747 \mathrm{~cm}^{-1}$. 
Supplementary Information

$\left[\mathbf{2}, \mathbf{4}, \mathbf{5}, \mathbf{6}, \mathbf{7}-\mathrm{D}_{\mathbf{5}}\right]$-Tryptamine $(\mathbf{2 4 a}) \cdot\left[2,4,5,6,7-\mathrm{D}_{5}\right]$-Tryptamine was synthesized from $\left[2,4,5,6,7-\mathrm{D}_{5}\right]-\mathrm{L}-$ tryptophan $(50.9 \mathrm{mg}, 0.24 \mathrm{mmol})$ as previously reported ${ }^{\mathrm{i}}$ for the nonlabeled compound to afford [2,4,5,6,7-D 5 -tryptamine $(25.2 \mathrm{mg}, 0.15 \mathrm{mmol}, 62 \%) .{ }^{1} \mathrm{H}$ NMR (500 MHz, CD $\left.{ }_{3} \mathrm{OD}\right): \delta 2.90(\mathrm{~m}, 4 \mathrm{H})$. HRMS-EI $\mathrm{m} / z$ (\% relative abundance): measured 165.1315 (20), calculated for $[\mathrm{M}]^{+}\left(\mathrm{C}_{10} \mathrm{H}_{7} \mathrm{D}_{5} \mathrm{~N}_{2}\right) 135.0967$ (100). FTIR $v_{\max }$ : $3302,1670 \mathrm{~cm}^{-1}$.

Isalexin (9). HPLC $t_{\mathrm{R}}=3.7 \mathrm{~min} ;{ }^{1} \mathrm{H}$ NMR $\left(500 \mathrm{MHz}, \mathrm{CD}_{3} \mathrm{CN}\right): 8.82\left(\mathrm{br} \mathrm{s}, 1 \mathrm{H}, \mathrm{D}_{2} \mathrm{O}\right.$ exchangeable), $7.52(\mathrm{dd}, J=8,8 \mathrm{~Hz}, 1 \mathrm{H}), 6.67(\mathrm{~d}, J=8 \mathrm{~Hz}, 1 \mathrm{H}), 6.50(\mathrm{~d}, J=8 \mathrm{~Hz}, 1 \mathrm{H})$, 3.91 (s, 3H); ${ }^{13} \mathrm{C}$ NMR (125 MHz, CD $\left.{ }_{3} \mathrm{CN}\right): 181.7$ (s), 160.8 (s), 160.0 (s), 152.3 (s), 142.0 (d), 108.4 (d), 107.8 (s), 105.6 (d), 57.2 (q); HREIMS $m / z$ (\% relative abundance) measured: 177.0422 (177.0426 calcd for $\mathrm{C}_{9} \mathrm{H}_{7} \mathrm{NO}_{3}$ ); EIMS $m / z$ (\% relative abundance): $177[\mathrm{M}]^{+}(83), 149$ (100), 122 (35), 107 (42), 63 (9). FTIR $v_{\max }: 3321,1748,1717,1620$, 1601, 1493, 1244, 1101, $775 \mathrm{~cm}^{-1} . \mathrm{UV} \lambda_{\max }(\log \varepsilon) 199$ (4.2), 234 (3.9), 335 (3.4).

(') Hashimoto, M.; Eda, Y. Osanai, Y.; Iwai, T.; Aoki, S. Chem. Lett. 1986, 6, 893896. 